\title{
Obkircher, Stefan (2017): Raumentwicklung in Grenzregionen. Bedeutung und Wirkung von Planungsleitbildern und Governance-Prozessen
}

\author{
Bielefeld - Transcript Verlag. = Sozial- und Kulturgeographie 15. 38 Abb., 295 S.
}

\section{Florian Weber ${ }^{1} \mathbb{B}$}

Online publiziert: 3. Juli 2018

๑) Springer-Verlag GmbH Deutschland, ein Teil von Springer Nature 2018

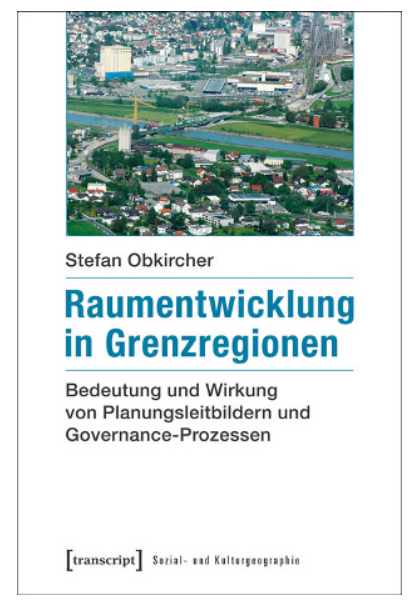

Wie steht es um die Entwicklung und praktische Gestaltung einer grenzüberschreitenden Zwischenstadt „Alpenrheintal"? So ließe sich vereinfacht die zentrale Frage der Veröffentlichung von Stefan Obkircher zu „Raumentwicklung in Grenzregionen“ zusammenfassen. Der Autor fokussiert das Vorarlberger und das St. Galler Rheintal in den Alpen als einen dynamisch sich entwickelnden Raum in Grenzlage, der bisher keiner ausführlichen Analyse unterzogen wurde, und setzt sich mit Herausforderungen grenzüberschreitender Planung und Zusammenarbeit auseinander. Er bewegt sich im Kontext einer Thematik, die allgemein vielfältige wissenschaftliche Bearbeitung erfährt (z. B. Paasi 1986; Wille/Reckinger/Kmec et al. 2016; Pal-

Dr. Florian Weber

florian.weber@uni-tuebingen.de

1 Forschungsbereich Geographie, Eberhard Karls Universität Tübingen, Rümelinstraße 19-23, 72070 Tübingen, Deutschland
lagst/Hartz/Caesar 2018), hier aber dezidiert auch praxisbezogen reflektiert wird.

Theoretisch-konzeptionell erfolgt mit der Arbeit eine Grundlegung über Raum- und Grenzkonstruktionsprozesse, die Terminologie der Zwischenstadt (Sieverts 1997) sowie die Debatten um Regional Governance. In dieser Konstellation ordnet Obkircher das so bezeichnete „Alpenrheintal“ 1) administrativ anhand von Vorarlberger und St. Galler Rheintalgemeinden, 2) als grenzüberschreitende Zwischenstadt, die heute durch ein Verwischen der Grenzen zwischen Stadt und Land in Zusammenhang mit einem Bevölkerungs- und Siedlungsflächenwachstum geprägt sei, sowie 3) als mentalen Raum aufgrund verschiedener Wahrnehmungen und somit auch alläglichen Regionalisierungen (vgl. Werlen 1997) ein. Ausgehend von einem sozialkonstruktivistischen und handlungsorientierten Raumverständnis werden Top-down-Leitbilder von Entscheidern und Planern den Bottom-up-Raumbildern (in Anlehnung an Ipsen 1997) von Bewohnern gegenübergestellt, um anwendungsbezogen Handlungsfelder grenzüberschreitender Entwicklung zugunsten einer ,grenzüberschreitenden Zwischenstadt" abzuleiten.

Die Kontrastierung basiert auf einem breit angelegten ,Werkzeugkasten“ aus quantitativen und vorrangig qualitativen Bestandteilen. Berücksichtigung finden statistische Daten einschließlich kartographischer Visualisierungen, Begehungen und Spaziergänge, teilnehmende Beobachtungen, Werkstattgespräche, explorative Straßenbefragungen, Interviews, visuelle Methoden wie mentale Landkarten und Photobefragung sowie vielfältige Dokumente zugunsten eines „State of the Art" ebenso wie ein Feldtagebuch.

Im Zusammenspiel entsteht so ein „Gesamtbild“ beziehungsweise ein gewisses „Mosaik“ der „Grenzregion Alpenrheintal“". Zunächst wird auf Planung und Governance bezogen herausgearbeitet, wie vor dem Hintergrund 
der identifizierten Herausforderung von Zersiedelung und dem Leitbild einer polyzentrischen Raumentwicklung unterschiedlich weitreichend grenzüberschreitend zusammengearbeitet wird, was besonders die beiden Fallstudien „Vision Rheintal“ und „Agglomerationsprogramm Rheintal“ verdeutlichen. Hier zeigen sich auch Unterschiede im Hinblick auf eher top down und eher bottom up ausgerichtete Ansätze sowie Beteiligung. Mit dem Fokus auf Raumbilder von Bewohnern wird sich im Verhältnis der tendenziell eher planungsbezogen distanziert betrachteten Zwischenstadt in ihrer alltagsweltlichen Wahrnehmung angenähert. Gerade mittels Zitatpassagen werden plastische Einordnungen im Spannungsverhältnis zwischen ländlichem Charakter und zunehmender Verstädterung im Wuchern, Wabern, übergangslosen Ineinandergreifen deutlich. Bedeutungszuweisungen fallen mehrdeutig aus, Spannungsfelder und Brüche werden sichtbar. Auf diese Weise erfährt die planungsbezogene Perspektive eine Erweiterung, die praxisbezogen nutzbar gemacht werden kann. So leitet Obkircher drei Handlungsfelder ab: 1) die Stärkung der grenzüberschreitenden Vernetzung mit einem polyzentrischen Leitbild, 2) die Institutionalisierung der Zusammenarbeit über projektbezogene Arbeit hinaus sowie 3) die stärkere Verankerung eines gemeinsamen Bewusstseins für Herausforderungen in der Region und damit ein aktives Leben einer „grenzüberschreitenden Zwischenstadt“.

Darauf aufbauend werden Denkanstöße für eine nachhaltige Raumentwicklung formuliert, die schließlich mit dem abgeglichen werden, was sich in den letzten Jahren abgezeichnet hat. Neben gewissen Konsistenzen wie einer stärkeren administrativen Berücksichtigung des wahrgenommenen Raumes zeigt sich nach Obkircher im Hinblick auf die Thematik der Zwischenstadt, dass diese im Sprachgebrauch und in der Planungspraxis bisher keine Verankerung erfahren habe. Wie eine „,neue Grenzkultur" schließlich aussehen könne, müsse sich wiederum erst noch in den kommenden Jahren zeigen.

Die resümierenden Ausführungen haben bereits deutlich gemacht, dass Stefan Obkircher eine ausführliche Analyse des regionalen Kontextes "Alpenrheintal“ Vorarlberg/St. Gallen gelungen ist. Dies ist einer langjährigen tiefgehenden Aufarbeitung einerseits und einer Tätigkeit in der Praxis als Grundlage für eine Relationierung von wissenschaftlicher Analyse und anwendungsbezogener Bewertung andererseits zu verdanken. Eine große Stärke des Buches liegt in der Innenperspektive und der aktiven Reflexion des Autors zwischen seinen Rollen als Forschender und Praktiker, was besonders auch das letzte Kapitel eines Abgleichs zwischen abgeleiteten Denkanstößen und zwischenzeitlichen Entwicklungen unterstreicht. Für die Zusammenarbeit über administrative Grenzen hinweg ergeben sich vielfältige Hinweise und Ansatzpunkte. Darüber hinaus zeigt der Autor sehr schön, dass es gelingen kann, trotz unterschied- licher theoretischer und methodischer Bausteine nicht den Überblick zu verlieren: Ein Kurzüberblick zu Beginn gibt Halt und Orientierung, der Aufbau der Veröffentlichung ist klar nachvollziehbar, die Kapitel bauen logisch aufeinander auf, die Ergebnisdarstellung erfolgt in sprachlich sehr guter Lesbarkeit in Verbindung mit plastischen Zitaten.

Mit „Raumentwicklung in Grenzregionen. Bedeutung und Wirkung von Planungsleitbildern und GovernanceProzessen“ ist aus meiner Perspektive der Titel allerdings etwas weit gegriffen, da konkret ein Ausschnitt der Grenzregion Schweiz/Liechtenstein/Österreich untersucht wird und keine Relationierung zu anderen Grenzregionen erfolgt. Eine gewisse Herausforderung stellt auch die Terminologie der „Zwischenstadt“ dar. Der Autor merkt selbst an, dass diese bis heute durchaus eher negativ konnotiert sei und planungsbezogen bis heute wenig dazu diente, in Wert gesetzt zu werden. Hier hätte sich im Zuge von Uneindeutigkeiten und Hybriditäten gegebenenfalls eher ein Rückgriff auf das Konzept der StadtLandHybride nach Kühne $(2012,2018)$ angeboten, das in der Vielgestaltigkeit und der Pasticheartigkeit konstitutive Bestandteile der Postmoderne sieht und somit nicht versucht, nach Eindeutigkeiten zu suchen, die im Verwischen begriffen sind. Im Hinblick auf die Etablierung grenzüberschreitender Entwicklung und einer Regionskonstitution wäre wiederum ein Aufgreifen der „stages“ der Regionalisierung nach Paasi (1986) denkbar gewesen, um einzuordnen, wo das „Alpenrheintal“ - praxisbezogen und handlungsorientiert entkomplexisierend gerade steht. Schließlich fällt auf, dass zwar Photovoice als Methode zum Einsatz kam, aber Fotografien von Befragten nicht im Buch aktiv zu finden sind. Da der Zugang noch nicht als weitreichend etabliert bezeichnet werden kann, hätte hier eine Verknüpfung aus Aufnahmen und Interviewsequenzen noch tiefergehenden Mehrwert erzielen können. Die angerissenen Kritikpunkte sind aber eher als Alternativoptionen anzusehen. In der Gesamtschau liegt eine Veröffentlichung vor, die Grenzraumforschern, Planern, Praktikern sowie gerade auch Politikern sehr empfohlen sei, um sich multiperspektivisch komplexen Herausforderungen im 21. Jahrhundert zwischen politisch-planerischer Steuerung und Lebenswelt anzunähern.

\section{Literatur}

Ipsen, D. (1997): Raumbilder. Kultur und Ökonomie räumlicher Entwicklung. Pfaffenweiler. $=$ Stadt, Raum und Gesellschaft 8.

Kühne, O. (2012): Stadt - Landschaft - Hybridität. Ästhetische Bezüge im postmodernen Los Angeles mit seinen modernen Persistenzen. Wiesbaden.

Kühne, O. (2018): Landschaftstheorie und Landschaftspraxis. Eine Einführung aus sozialkonstruktivistischer Perspektive. Wiesbaden.

Paasi, A. (1986): The institutionalization of regions: a theoretical framework for understanding the emergence of regions and the 
constitution of regional identity. In: Fennia - International Journal of Geography 164, 1, 105-146.

Pallagst, K.; Hartz, A.; Caesar, B. (Hrsg.) (2018): Border Futures Zukunft Grenze - Avenir Frontière. Zukunftsfähigkeit grenzüberschreitender Zusammenarbeit. Hannover. = Arbeitsberichte der ARL 20.

Sieverts, T. (1997): Zwischenstadt. Zwischen Ort und Welt, Raum und Zeit, Stadt und Land. Gütersloh, Berlin, Basel, Boston. = Bauwelt-Fundamente 118
Werlen, B. (1997): Sozialgeographie alltäglicher Regionalisierungen. Band 2 Globalisierung, Region und Regionalisierung. Stuttgart. = Erdkundliches Wissen 119.

Wille, C.; Reckinger, R.; Kmec, S.; Hesse, M. (Hrsg.) (2016): Spaces and Identities in Border Regions. Politics - Media - Subjects. Bielefeld. 